\title{
Research on the Construction Method of Public Security Map based on Emergency Space Big Data
}

\author{
Xuerui Chen ${ }^{1, \mathrm{a}}$, Long Chen ${ }^{1, \mathrm{~b}}$ \\ ${ }^{1}$ Tsinghua University, Public Safety Institute, Department of Engineering Physics, Beijing, China
}

\begin{abstract}
In view of the current problems encountered in public safety and emergency management, this study proposes a method for constructing a public safety map. Based on the public safety theory and the existing POI system, a method for constructing a public safety attribute index system is proposed, and a preliminary discussion is made on the collection mechanism of public safety attribute data and the operation mechanism of the public safety map. It is hoped to provide a universal and easy access to emergency space information for emergency management departments and rescue workers.
\end{abstract}

\section{Introduction}

China is one of the countries with the most serious disasters in the world, and emergencies are prone to occur frequently. According to statistics, in 2018, natural disasters nationwide caused more than 136 million people to be affected, resulting in more than 200 billion yuan in direct economic losses [1], and more than 29,500 deaths as a result of various work safety accidents [2]. In emergency management, more than 80 percent of the information is geospatial information. Therefore, geographic information technology plays an irreplaceable role in emergency management. Current geographic information in the emergency management is applied to professional auxiliary decision-making and emergency rescue systems, has the high professional. At the same time, professional systems require high professional quality of users, which makes it impossible for on-site rescue workers with different skill levels to quickly obtain basic spatial information and safety information of disaster objects around the event.

Based on the emergency big data and guided by the public security triangle theory, this research studied and discussed the key links and technologies of building the public security map based on the POI system.

\section{Connotation of public safety map}

Under the guidance of the triangle theory of public security, the public security map proposed in this study takes the POI system as the starting point and comprehensively considers the original function and utility between disaster victims and emergencies. Based on the research on the extraction method of security attribute index of various POI, the data update mechanism and user application mechanism are formulated to form the public security map with security attribute. Public security map not only enables ordinary commanders and fighters to quickly grasp all kinds of basic security elements of disaster objects, improve rescue efficiency, avoid rescue errors, and reduce casualties; Moreover, it can provide the spatial information base layer framework with public security attribute for the construction of various professional business systems.

To sum up, the construction of public security map is mainly divided into two important links: POI security attribute index system construction method research, data construction and application mechanism research. Each link in Figure 1 will be described in detail below.

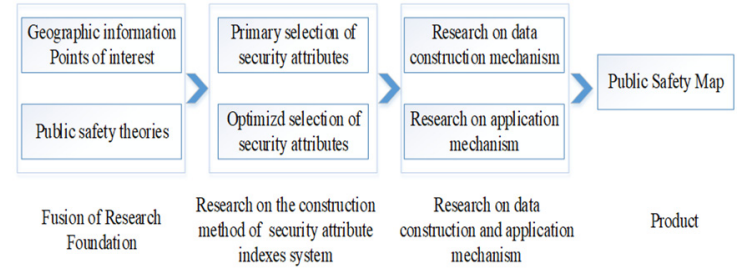

Fig1. Construction method of public safety map

\section{Theoretical basis and research status}

\subsection{Public Safety Triangle Theory and POI}

The triangle theory of public safety holds that the framework of public safety technology can be represented by a triangle whose three sides respectively represent emergencies, disaster objects and emergency management. Nodes connecting three sides are collectively called disaster elements, which respectively include material, energy, and information. In essence, disaster elements are an objective existence, when these disaster elements exceed the critical quantity or meet certain trigger conditions, they may lead to an emergency, and do not cause damage before the critical quantity or trigger is not

achenxueruiabc@163.com, bchenlongbuaa@126.com 
exceeded $[3,4]$.

According to national standards, POI (points of interest) is mainly divided into 14 categories, including catering, accommodation, health and social security, science and education and culture, sports and leisure, and tourist attractions etc. [5] At present, POI is widely used in electronic maps, such as Baidu Map, AutoNavi map, Tencent map, which are widely used and recognized. However, the focus of electronic maps is more on navigation and path planning, and the public safety attributes of POI are not involved.

\subsection{Research Status}

At present, there are few researches on the public security map which can serve the public, rescue workers, and serve as the foundation for the construction of professional business system. At present, there are many researches on regional security and crime map. The United States, the United Kingdom, South Korea, and other countries have also carried out relevant research. South Korea has built a "National Life security Map", marking crime, traffic accidents, natural disasters, and other information on the map. According to this information, all walks of life and local governments can participate in activities to improve regional security. Police in The US city of New York have released a crime map that identifies areas of danger by colors and provides the ability to plan safe routes around danger areas. The UK also provides information about where and when crimes are committed, depending on the level of crime. Although the study of these public security indexes and maps considers the elements of public security, there is no in-depth study on the attributes of public security, such as the vulnerability, risk, resource, and capacity of disaster-bearing bodies.

\section{The key links of public security map construction}

\subsection{Research on the Construction Method of Public Security Attribute Index System}

Because there are many different kinds of POI, class differences between the index and complex, and the map to ensure public safety in public security attribute information of each type of POI completeness, systematic, typicality, practicality and other characteristics, this study puts forward an index system of public security map building method, used in the selection of all kinds of security attribute index of the POI.

According to the triangle theory of public security, most of the POI are hazard-affected bodies with four attributes: hazard, resource, vulnerability, ability. Hazard refers to the possibility that the affected objects itself causes an emergency and the risk that the hazard-affected bodies will bring losses to the surrounding environment or other hazard-affected bodies after the occurrence of an emergency. Resource refers to the ability of the hazardaffected bodies to provide emergency resources to the outside world after an emergency occurs. Vulnerability refers to the possibility and degree of damage suffered by the disaster-bearing bodies based on its physical characteristics in an emergency $[6,7,8]$. Capability refers to the ability to cope with emergencies and reduce losses, as well as the ability to rebuild and repair.

\subsubsection{Preliminary election of $\mathrm{POI}$ security attribute indexes}

Combined with the characteristics of hazard-affected bodies in public safety and emergency management, the method of literature analysis and expert research was used to conduct the primary selection of POI safety attribute index, forming a 3-level POI safety attribute index system as shown in Figure 2. The hierarchical framework of the index system mainly includes the target layer, the criterion layer and the operation layer. The target layer (GI) is the expression level of public safety attributes. The criterion layer (AI) in this study refers to the public safety characteristics of POI. The operation layer (CI) represents specific indicators.

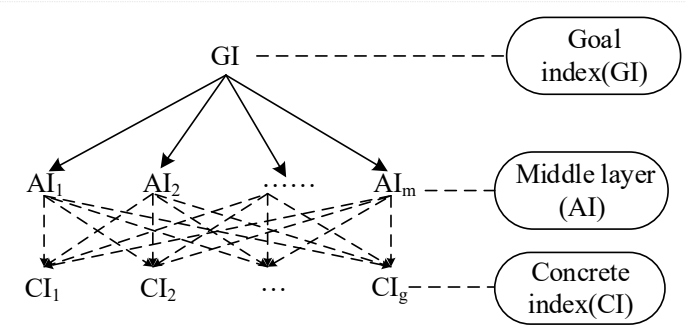

Fig2. Hierarchy structure of indexes system

In this study eight criteria for choosing single index were pointed out, following the theory of usability engineering, the method of economic theory and system analysis. The eight criteria are $M$ (measurability), $\mathrm{V}$ (vulnerability), $\mathrm{P}$ (predictability), $\mathrm{T}$ (typicality), C(controllability), I(integrity), R(responsibility) and $\mathrm{S}$ (stability). Among these criteria, I is used to restrict the building of index system, while the other 7 criteria play their roles in the choosing process of single index.

\subsubsection{An optimization model for index selection}

The quantitative method is adopted to optimize the selection of POI security attribute indexes, and the construction of index system that can reflect POI public security attribute is realized by constructing relation matrix, constructing standard degree matrix, and establishing objective function.

a) Construction of relation matrix

In the basis of the hierarchy framework of POI public security attribute index system, a relationship matrix $R$ were built. The matrix $\mathrm{R}$ represents the relation between indexes of layer $n$ and indexes of layer $n-1$.

$j$ indicates the $\mathrm{j}$ index in the layer $n-1$,

$g$ indicates the $\mathrm{j}$ index in the layer $n$,

$$
\mathrm{A}=\left[\begin{array}{ccc}
\mathrm{a}_{11} & \cdots & \mathrm{a}_{1 \mathrm{~g}} \\
\vdots & \ddots & \vdots \\
\mathrm{a}_{\mathrm{j} 1} & \cdots & \mathrm{a}_{\mathrm{jg}}
\end{array}\right]
$$

$\mathrm{r}_{j g}=1$ means $g$ is associated with $j$,

$\mathrm{r}_{j g}=0$ means there is no connection between $\mathrm{g}$ and $j$.

b) Construction of selection criteria matrix 
Following the principle of index selection criteria set above, for the selection of a single index, a selection matrix B $(g \times 7)$ of $g$ rows and 7 columns is constructed.

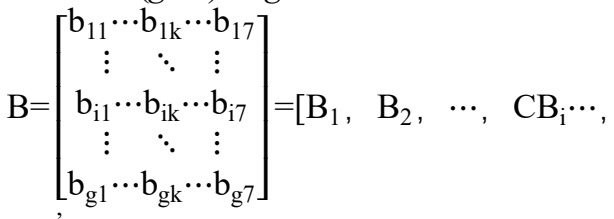$$
\left.\mathrm{B}_{\mathrm{g}}\right]
$$

$g$ is the number of indexes in layer $n$,

$i$ and $k$ respectively represent the $i$ index and the $k$ criteria,

$c_{i k}=1$ means the index could meet the criteria,

$c_{i k}=0$ means the index could not meet the criteria.

c) Quantitative optimization of index system indicators

A perfect index system must not only have index integrity but also ensure the simplicity of the system. Therefore, in order to build a perfect POI security attribute index system, based on the relationship matrix and the standard degree matrix, an objective function optimization index system is built. The function is as follows.

$$
\min z=\sum_{m=1}^{g} c i_{m}
$$

At the same time, the objective function meets certain constraints: if the evaluation index meets the 5 evaluation criteria set above, it is directly selected, and its mathematical expression is as follows.

$$
c i_{m}=1 \text { if } M \times B_{m}^{T} \geq 5
$$

Among them, $c i_{m}=1$ means that the specific index $\mathrm{m}$ of the operation layer is selected. If $M \times B_{m}{ }^{t}<5$, then the index $m$ is not selected, and $c i_{m}=0$.

By constructing the vector $C I=\left[c i_{1}, c i_{2}, \ldots, c i_{g}\right]$ to ensure the correlation between the indicator layers, the vector CI needs to meet the following conditions.

$$
\mathrm{CI} \times \mathrm{A}^{\mathrm{T}}>0
$$

Completeness is an important indicator of a perfect indicator system. According to the requirements of the principal component analysis method, 0.85 is selected as the completeness standard of the indicator system. Therefore, the sum of the weights of the indicator system cannot be less than 0.85 , as shown in the following formula.

$$
\mathrm{CI} \times \mathrm{W}^{\mathrm{T} i}{ } \geqslant 0.85
$$

Where $\mathrm{W}^{\mathrm{T}}$ is a matrix composed of the weights of all specific indicators.

\subsection{Research on Construction and Application Mechanism of Public Safety Map}

\subsubsection{Data construction and update mechanism}

The data of POI IN public security map is very different from that in electronic map. The data has the characteristics of strong professionalism, wide variety, and wide range of coverage. Therefore, it is necessary for multiple parties to participate in the construction and maintenance of data, to ensure the freshness of data. The collection and realization of public security map data needs the participation of government departments, geographic information service providers, emergency service providers and the public.

In accordance with the international and domestic industry standards and software engineering standards, the establishment of a sound standard system, which includes unified standards for spatial data, service standards, information expression standards, and security standards. These standards ensure the interconnection, information sharing, coordinated operation, security and reliability of the platform and its functional systems. Starting from the business scenario of public safety and emergency management, the research and development of public safety map with reasonable structure, practical function and support actual business needs is promoted.

Through a complete set of data management, data exchange, data interface specifications to achieve data update and maintenance. At the same time, according to the principle of "who collects, who maintains, who is responsible and who authorizes", paid collaboration can be established between the government, emergency service providers and spatial information producers. Public security attribute data needs to be obtained from multiple channels to ensure the timeliness of the data. The freshness of data is the guarantee for the promotion and application of public safety maps, and it will also help to form an ecosystem around public safety maps.

Based on the unified indicator system and standards and norms, departments and organizations at all levels are encouraged to conduct data collection and release and research and development of application services based on public security maps. To form an orderly co-construction and sharing mechanism for public security maps. More emergency developers, geographic information service providers and data manufacturers can participate in the construction of public security maps by providing support and guarantee systems in standards, services and circulation and following the principles of openness, sharing, empowerment and win-win. To build a benign industry ecosystem of survival of the fittest with perfect guarantee mechanism, sufficient business demand, multiparty co-construction and sharing.

\subsubsection{Application Mechanism}

The main application objects of public security map are the public, government, enterprises. The application of public security map can also be divided into two modes: free and paid, among which free public security map can be provided to the public in the form of APP and web application. In case of an emergency, the public can obtain the location of fire facilities and refuge places through the public safety map and obtain certain self-help ability. At the same time, the rescue workers who rushed to the scene in the first time could quickly acquire the vulnerability caused by the disaster, and the preliminary study and judgment of the damage to the surrounding disaster victims could greatly reduce the loss of personnel and property caused by the lack of information.

Based on the public security map, the government and enterprises can carry out extended in-depth business 
application construction, which can serve the five business domains of supervision and management, monitoring and early warning, command and rescue, decision support and government affairs management.

\section{Conclusions}

In this study, a preliminary discussion was made on the construction method of public safety map. However, the construction of public safety maps not only requires a lot of data collection work, but also requires the joint participation of multiple parties to build a truly capable of serving public safety and emergency management. Therefore, how to build a public safety map based on new science and technology such as big data and cloud computing requires in-depth research. At the same time, a set of highly practical data update and application mechanisms need to be improved through practice.

\section{Acknowledgment}

The authors appreciate the subject for research and demonstration of key technologies for dynamic identification and early warning of safety risks in crowded places based on action trajectories (Z181100009018010), one of Beijing Science and Technology Plan.

\section{References}

1. National Bureau of Statistics of China. China Statistical Yearbook 2019, China Statistics Press,2019

2. National Bureau of Statistics of China. Statistical bulletin of national economic and social development of the people's Republic of China in 2019. http://www.stats.gov.cn/tjsj/zxfb/202002/t20200228 1728913.html.

3. F. Weicheng, L. Yi, W. Wenguo. "Triangular Framework and "4+1" Methodology for Public Security Science and Technology", Science \& Technology Review, March 2009.

4. L. Yi, N. Shunjiang, W. Wenguo, F. Weicheng. Development of the Public Safety System and a Security-Guaranteed Society, January,2017, pp. 118123.

5. Classification and coding of geographic information points of interest (GB/T 35648-2017). Administration of Quality Supervision, Inspection and Quarantine of the People's Republic of China and Standardization Administration of China,2017.

6. International Strategy for Disaster Reduction [EB/OL]. http://www.unisder.org/eng/library/libterminology-eng\%20home.htm,2008.

7. UNDP. Human development report: cultural liberty in today's diverse word[C].Now York: United Nations Development Program , 2004.

8. L. Guanhui, Z. Changyi. "Vulnerability after a Devastating Hazard: An Interpretation of Land Use and Land Cover Change in Central Taiwan Since 1999 Chi-Chi Earthquake", Advances in Earth
Science, vol.21, pp.201-210, February 2006. 